\title{
Erratum to: Involvement of $\beta$-arrestins in cancer progression
}

\author{
Shanshan Hu $\cdot$ Di Wang $\cdot$ Jingjing Wu \\ Juan Jin $\cdot$ Wei Wei $\cdot$ Wuyi Sun
}

Published online: 1 December 2012

(C) Springer Science+Business Media Dordrecht 2012

\section{Erratum to: Mol Biol Rep}

\section{DOI 10.1007/s11033-012-2148-0}

The original article has been published incorrectly with errors in the corresponding author names. Wuyi Sun and Wei Wei is the corresponding author of this article. The correct corresponding author name is given below.

The online version of the original article can be found under doi:10.1007/s11033-012-2148-0.

S. Hu · D. Wang · J. Wu · J. Jin · W. Wei $(\bowtie) \cdot$ W. Sun $(\bowtie)$ Institute of Clinical Pharmacology, Anhui Medical University,

Key Laboratory of Anti-inflammatory and Immunodrugs,

Education Ministry of China, Hefei 230032, Anhui Province,

People's Republic of China

e-mail: wwei@ahmu.edu.cn

W. Sun

e-mail: sunwuyi51@yahoo.com.cn

$\mathrm{S} . \mathrm{Hu}$

e-mail: shanshanhu1986@yahoo.com.cn 